\title{
Erratum
}

\section{Miscellany on traces in $\ell$-adic cohomology: a survey}

\section{Luc Illusie}

Received: 23 March 2007

Published online: 21 September 2007

(C) The Mathematical Society of Japan and Springer 2007

Communicated by: Takeshi Saito

Japan. J. Math. (2006) DOI 10.1007/s11537-006-0504-3

Theorem 2.3 is correct as stated. However, in Lemma 7.1, in addition to the other hypotheses, $S$ should be assumed geometrically unibranch. Otherwise the implication $(1) \Rightarrow(2)$ may not hold. Indeed, Gabber gave the following example. Let $S$ be the $\mathbb{F}_{p}$-scheme obtained from the affine line over $\mathbb{F}_{p}$ by identification of the points 0 and 1 . By definition, $S=\operatorname{Spec} A$, where $A$ is the subring of $\mathbb{F}_{p}[t]$ consisting of the polynomials $P$ such that $P(0)=P(1): S$ is the cubic in $\mathbb{A}_{\mathbb{F}_{p}}^{2}=$ $\operatorname{Spec} \mathbb{F}_{p}[x, y]$ with equation $x^{3}-y^{2}+x y=0$ (an isomorphism $\mathbb{F}_{p}[x, y] /\left(x^{3}-y^{2}+\right.$ $x y) \stackrel{\sim}{\longrightarrow} A$ is given by $\left.x \mapsto t^{2}-t, y \mapsto t\left(t^{2}-t\right)\right)$. Let $Z$ be the connected étale cover of degree 2 of $S$ which splits over the normalization of $S$. With the notations of Lemma 7.1, take $a=2$ and $n \geq 1$ such that $\ell^{n}>2$. Let $g \in \pi_{1}(S, \bar{\eta})$ correspond to the involution permuting the sheets of $Z$. Then $|Z(s)|=a=2$ for every point $s$ of $S$ with value in a finite field. However, $\operatorname{Tr}\left(g, H_{c}^{*}\left(Z_{\bar{\eta}}, \mathbb{Q}_{\ell}\right)\right)=0$, and $0 \neq 2$ in $\mathbb{Z} / \ell^{n} \mathbb{Z}$. So (1) does not imply (2) in this case. If $S$ is assumed geometrically unibranch, then the proof given in loc. cit. is correct (the extra assumption is

L. ILLUSIE

Département de Mathématiques, Bâtiment 425, Faculté des Sciences d'Orsay, Université ParisSud 11, F-91405 Orsay Cedex, France

(e-mail: luc.illusie@math.u-psud.fr)

The original article was published in the Japanese Journal of Mathematics, volume 1 number 1, p.107-p.136. Its online version can be found at http://dx.doi.org/10.1007/s11537-006-0504-3 
used in the application of Čebotarev's theorem). On the other hand, the proof of 2.3 given in 7.2 is correct, as the implication (1) $\Rightarrow(2)$ of 7.1 is used only in the case where $S$ is normal.

In 7.1, for a $\mathbb{Q}_{\ell}$-sheaf $\mathscr{F}$ on $S$, lisse is tacitly assumed to mean that $\mathscr{F}$ corresponds to a continuous representation of $\pi_{1}(S, \bar{\eta})$ in a finite dimensional $\mathbb{Q}_{\ell^{-}}$ vector space, in other words, $\mathscr{F}=\mathscr{F}_{0} \otimes \mathbb{Q}_{\ell}$, for a lisse $\mathbb{Z}_{\ell}$-sheaf $\mathscr{F}_{0}$ on $S$. It is well known, however, that if $S$ is not geometrically unibranch, there may exist $\mathbb{Q}_{\ell}$-sheaves on $S$ which are lisse in the sense of [D2, 1.1.1], i.e., are étale locally of the form $\mathscr{F}_{0} \otimes \mathbb{Q}_{\ell}$ for a lisse $\mathbb{Z}_{\ell}$-sheaf $\mathscr{F}_{0}$, but are not globally of this form (contrary to what is suggested in [D2, 1.1.6]). I learnt the following example from Gabber. Take $S=\operatorname{Spec} A$ as above. Let $Y$ be the $S$-scheme deduced from $\left(\mathbb{A}^{1} \times \mathbb{G}_{m}\right)_{\mathbb{F}_{p}}$ by identifying $\{0\} \times x$ with $\{1\} \times x^{\ell}$ (in other words, $Y=\operatorname{Spec} B$, where $B$ is the subring of $\mathbb{F}_{p}\left[t, s, s^{-1}\right]$ consisting of the elements $P$ such that $\left.P(0, s)=P\left(1, s^{\ell}\right)\right)$. Let $f: Y \rightarrow S$ be the projection; this is a morphism of finite type, with fibers isomorphic to $\mathbb{G}_{m}$. The $\mathbb{Q}_{\ell}$-sheaf $\mathscr{F}:=R^{2} f_{!} \mathbb{Q}_{\ell}$ on $S$ is lisse of rank 1 in the sense of [D2, 1.1.1], but does not come from a lisse $\mathbb{Z}_{\ell}$-sheaf on $S$ (if $\pi: \mathbb{A}_{\mathbb{F}_{p}}^{1} \rightarrow S$ is the normalization of $S$, and $i:\{0\} \rightarrow S$ the inclusion of the double point, $\mathscr{F}$ is the kernel of the map $\pi_{*} \mathbb{Q}_{\ell}(-1) \rightarrow i_{*} \mathbb{Q}_{\ell}(-1)$ defined by $(1,-\ell)$ at the double point).

page 134, line 9: $A$ is not the sub- $k$-algebra $B$ of $K$ generated by $Y$ and $X Y^{-n}$ for $n \geq 1$, but a localization of $B$. This error, however, is of no consequence for the rest of the remark.

Acknowledgements. I thank Gabber heartily for the examples. I am also grateful to Deligne for useful discussions and to T. Saito for comments and suggestions on a first draft.

This note was prepared while I was visiting the Institute for Advanced Study in Princeton, supported by a grant from the Oswald Veblen Fund. I wish to express my deep gratitude to this institution for its warm hospitality and generous support. 